\title{
Interlocking Numerical Taxonomies ${ }^{1}$
}

\author{
L. R. HILL \\ National Collection of Type Cultures, Central Public Health Laboratory, London, United Kingdom
}

The question is asked, Has the application of numerical taxonomy in microbiology been sufficiently extensive to permit the construction of a general classification of bacteria from exclusively numerical taxonomy data? A semiquantitative method of pooling (or interlocking) the results of numerical taxonomies was applied to three wide-ranging studies, using concepts of "specific" and "generalized" taxonomic spaces. Difficulties of the method are discussed and some alternative approaches to the problem are outlined.

Numerical taxonomy has made noteworthy progress in microbiology, and considerable taxonomic order has followed in many generic areas. A general problem is, however, whether anything can be done collectively with all the various numerical taxonomies that have been carried out. There are probably several different ways of approaching this problem. The method of interlocking numerical taxonomies described below is a crude, imperfect method, and it is presented not so much as a solution to the problem but rather as a means of drawing attention to the problem itself.

There are two facets to the problem. Firstly, a criticism is sometimes leveled at the practice of numerical taxonomy that it is not as objective as its proponents claim. The numerical taxonomist claims to assess objectively the similarities between strains of bacteria through not introducing any deliberate a priori bias in choice of operational taxonomic units (OTUs, i.e., strains) and tests and through unbiased mathematical and statistical methods. He claims to operate without a priori prejudices regarding the classification of those OTUs. But, so the criticism runs, the very selection of OTUs and tests for study destroys, at least partially, at the outset the claimed objectivity. No taxonomist, numerical or otherwise, can study as many strains in as many tests he would like to use. Therefore, limitations are set and usually not only on the number of strains to be studied, but also their kind (e.g., gram-positive cocci, aerobic spore-bearers, etc.). Having made that choice of OTUs, in turn, he determines to some extent the kinds of tests that will be used. Within these limitations the practice of numerical taxonomy may well be "objective," but the limitations themselves prevent a complete objectivity.

\footnotetext{
1 Text of a paper read at the XIth Conference on the Taxonomy of Bacteria, Brno. Czechoslovakia, 23 to 25 September 1974 .
}

The other facet to the problem is more mundane. Numerical taxonomy has had 15 years of widespread application in microbiology. It can claim, with justification, more success in microbiology than in any other biological field. Although not completely supplanting nonnumerical methods, it has become a most familiar, if not routine, taxonomic method in microbiology. There are few bacterial genera and species left untouched by numerical taxonomy. Is it not time, therefore, to ask the question, Has numerical taxonomy been applied in microbiology to a sufficiently wide extent to permit the construction of a general classification of bacteria from exclusively numerical taxonomy data and which would thus be independent of nonnumerical classification? If that question were answered positively, the criticism of a lack of complete objectivity in numerical taxonomy would be removed or considerably weakened.

In view of the amount of published numerical taxonomy already available, one of several possible lines of enquiry would be to devise a method of combining or pooling the different taxonomies, which is what the method of interlocking numerical taxonomies attempts to do.

\section{SPECIFIC AND GENERALIZED TAX- ONOMIC SPACES}

If separate numerical taxonomies are not wholly objective, it could be argued that their shortcomings will be carried over into any combination of them, and the pooled result will also not be wholly objective. I propose the following counterargument. In any one study, the OTUs are related to each other in a multidimensional space, the number of dimensions of which is the number of characters used to code the results data. The numerical taxonomy result, usually a dendrogram, shows the overall similarities between the OTUs relative to each other. The taxonomic space, therefore, is a 
well-defined and limited space determined by the particular sets of tests and OTUs, a "specific" taxonomic space. If different numerical taxonomies have some OTUs common to each, then their respective specific taxonomic spaces will overlap. There will be overlaps and exclusiveness both in OTUs and tests between the studies. If the different studies are somehow pooled, the resulting taxonomic space would be less well-defined but, at the same time, less limited (in other words, a "generalized" taxonomic space). This space will be less welldefined because, although the overlap in terms of OTUs can be precisely defined, this is not so regarding the tests for two reasons: first, the well-known lack of reproducibility of tests between laboratories. Second, similarities between OTUs are usually expressed through a coefficient (e.g., \%M) from which it is not possible to discover how much of the similarity lies in tests at least nominally overlapping and how much resides in nonoverlapping tests. The combined space will, however, be more general because it will contain more OTUs, related through a greater number of dimensions (illdefined as they may be), than any of the contributing specific taxonomic spaces on their own.

Regarding those OTUs common to more than one study, the different studies will give different similarity values between them. Similarity coefficients, however, are only estimates of the "true" phenetic similarity that would only be obtained if the complete phenotypic expressions of the genomes of the OTUs were known. Averaging similarity values from different studies yields simply an average estimate. In view of the overlapping, but not identical, sets of tests used in the different studies, an average estimate should be yet closer to the true similarity than any one estimate on its own.

The underlying assumption of the method of interlocking taxonomies is that it will achieve a gain in generality (a gain towards greater objectivity) that outweighs the limited objectivity of the contributing specific taxonomic spaces. (A similar assumption is the basis of the so-called "permissive" philosophy, used successfully by the International Working Group on Mycobacterial Taxonomy, for cooperative studies $[3,8]$.)

Sokal and Rohlf (7) introduced "co-phenetic values" which are useful to compare dendrograms. A dendrogram is divided into similarity classes by drawing phenon-lines across it at right angles to the similarity scale. Arbitrary numbers are then given to the similarity classes, and the co-phenetic value between a pair of OTUs is the arbitrary class number in which the two OTUs are connected in the dendrogram. From examination of a dendrogram divided in this manner, a triangular similarity matrix can be constructed and will contain co-phenetic values between all possible pairs of OTUs. The co-phenetic matrix can then be compared with the original similarity matrix, or with co-phenetic matrices obtained from other dendrograms, by means of co-phenetic correlation coefficients. Lockhart (4) used this method to compare classifications in which there was some overlap in OTUs. The data he used had all originated from his own laboratory, and the objective was to study the reproducibility of numerical taxonomies. Although co-phenetic values are rather like the transformed, standardized similarity values used in the present method of interlocking numerical taxonomies (see Method and Application), co-phenetic values have not been used to combine different numerical taxonomies.

\section{METHOD AND APPLICATION}

The method first isolates OTUs common to the different studies, extracts their pair-wise similarities as given by the different studies, standardizes these similarities, averages the similarities and, finally, uses the averaged standardized similarities to obtain a dendrogram intended to show the pooled result. The method has been applied to three wide-ranging numerical taxonomies, and details of the proposed method will emerge as the results are given.

The three studies chosen were those of widest range $(1,2,6)$ in the hope that the pooled result would indicate the major groupings; the method could then be applied within these major groupings with other numerical studies pertinent to them.

First, those OTUs common to at least two of the studies, and identifiably identical through their strain designations, were listed: some 14 OTUs (Table 1). For each study separately, a reduced similarity matrix was drawn up containing just those OTUs from the list of 14 and the similarities between them specific to the particular study. The actual numerical values of these extracted similarities cannot be directly averaged since they are related to their own specific taxonomic spaces and for other reasons (vide infra); they need to be standardized before averaging. A simple transformation was used (Fig. 1), taking the lowest value in the separate, reduced similarity matrices to equal 1 on an arbitrary scale, the highest value to equal 9 , and intermediate values given appropriate new values in the 1 to 9 arbitrary scale.

Next, a composite matrix was drawn up (part 
TABLE 1. OTUs obtained from the literature for phase I (identical OTUs, numbered 1 to 14 in column 2) and phase II (nonidentical OTUs, unnumbered in column 2) dendrograms ${ }^{a}$

\begin{tabular}{|c|c|c|c|c|}
\hline \multirow[b]{2}{*}{ Name } & \multirow{2}{*}{ OTUno. } & \multicolumn{3}{|c|}{ Literature source } \\
\hline & & $\begin{array}{l}\text { Sneath and } \\
\text { Cowan }(6)\end{array}$ & $\begin{array}{c}\text { Focht and } \\
\text { Lockhart (1) }\end{array}$ & $\begin{array}{l}\text { Goodfellow } \\
\text { (2) }\end{array}$ \\
\hline $\begin{array}{l}\text { Micrococcus luteus } \\
\text { Staphylococcus aureus } \\
\text { Salmonella choleraesuis } \\
\text { Escherichia coli } \\
\text { E. coli } \\
\text { Serratia marcescens } \\
\text { S. marcescens } \\
\text { S. marcescens } \\
\text { S. marcescens } \\
\text { Shigella dysenteriae } \\
\text { Pseudomonas aeruginosa } \\
\text { P. aeruginosa } \\
\text { P. aeruginosa } \\
\text { Bacillus subtilis } \\
\text { Staphylococcus afermentans } \\
\text { Bacillus cereus } \\
\text { B. cereus } \\
\text { B. pantothenticus } \\
\text { Chromobacterium violaceum } \\
\text { Mycobacterium phlei } \\
\text { Nocardia farcinica } \\
\text { Proteus vulgaris } \\
\text { P. vulgaris } \\
\text { Aeromonas hydrophila } \\
\text { Enterobacter cloacae } \\
\text { E. cloacae } \\
\text { Micrococcus roseus } \\
\text { Xanthomonas hyacinthi } \\
\text { X. hyacinthi }\end{array}$ & $\begin{array}{r}9 \\
10 \\
11 \\
12\end{array}$ & $\begin{array}{l}\text { NCTC } 4837 \\
\text { NCTC } 6750 \\
\text { NCTC } 2000 \\
\text { NCTC } 3610 \\
\text { NCTC } 2665 \\
\text { NCTC } 7464 \\
\\
\text { NCTC } 8162 \\
\text { NCTC } 9757 \\
\text { NCTC } 8151 \\
\text { NCTC } 4524 \\
\text { NCTC } 4636\end{array}$ & $\begin{array}{l}\text { ATCC } 398 \\
\text { ATCC } 12600 \\
\text { ATCC } 13312 \\
\text { ATCC } 11775 \\
\\
\text { ATCC } 13880 \\
\text { ATCC } 13313 \\
\text { ATCC } 10145 \\
\text { ATCC } 6051\end{array}$ & $\begin{array}{lr}\text { NCTC } & 10332 \\
\text { NCTC } & 3610 \\
\text { NCTC } & 2665 \\
& \\
\text { NCTC } & 7587 \\
\text { NCTC } & 8162 \\
\text { NCTC } & 9757 \\
\text { NCTC } & 8151 \\
\text { NCTC } & 4524 \\
& \\
\text { NCTC } & 7810 \\
& \\
\text { NCIB } & 8529 \\
\text { NCIB } & 7520 \\
& \\
\text { NCPPB } & 599\end{array}$ \\
\hline
\end{tabular}

a Abbreviations: ATCC, American Type Culture Collection, Rockville, Md.; NCIB, National Collection of Industrial Bacteria, Aberdeen, Scotland; NCPPB, National Collection of Plant Pathogeneic Bacteria, Harpenden, England; NCTC, National Collection of Type Cultures, London, England.

b Now renamed Micrococcus luteus.

of which is shown in Fig. 2), entering in each box the various similarity values (in arbitrary units) obtained from the different studies and then simply averaging the contents of each box. The method then proceeds to a standard clustering procedure-single linkage to obtain a first reordering of OTUs and finally construction of the composite dendrogram by average linkage (Fig. 3). This "phase I" dendrogram contains only identifiably identical OTUs common to at least two of the contributing studies.

If the combining process were limited to only identifiably identical OTUs, then the resulting taxonomy would be very limited, as in Fig. 3 . The next step is to consider those OTUs in the different studies which are similarly named but not identifiably identical. Adding these to the system is obviously contentious for it assumes they are indeed properly named and belong to a well-defined taxon. If the problem of combining numerical taxonomies is undertaken beginning with wide-ranging studies and proceeding later to narrower studies which involve just one or a few species (a from top-to-bottom strategy), then the contentiousness of adding nonidentical OTUs can be viewed in a perspective. In this first application, the contributing specific taxonomic spaces were themselves large, and an Escherichia coli in one study will be more like an $E$. coli in another study than to anything else in either study. This reasoning obviously leans heavily on a subjective estimate of the "goodness" of particular species and not all will be as simple as $E$. coli.

As with the identifiably identical OTUs, the similarly named, but not identical, OTUs in the three studies were listed (a further 15 OTUs, Table 1), and the similarities (in arbitrary units) between them and with the OTUs already included in the phase I dendrogram were 
Arbitrary scale

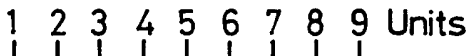

Sneath

\& Cowan

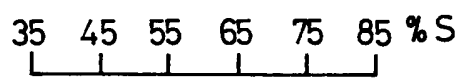

Focht \& Lockhart

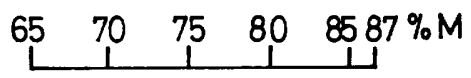

Goodfellow 74

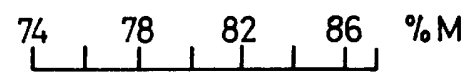

Fig. 1. Transformation of original $\% S$ and $\% M$ values to an arbitrary scale.

\begin{tabular}{|c|c|c|c|c|}
\hline & & 1 & 2 & 3 \\
\hline 1. M.luteus & & & & \\
\hline 2. S.aureus & $\begin{array}{l}a \\
b\end{array}$ & $\begin{array}{ll}5 \cdot 8 & \\
5 \cdot 4 & 5 \cdot 6 \\
\end{array}$ & & \\
\hline 3. E.coli & $\begin{array}{l}a \\
b\end{array}$ & $\begin{array}{ll}2 \cdot 6 & \overline{4 \cdot 0} \\
5 \cdot 4 & 4\end{array}$ & $\begin{array}{l}4 \cdot 2 \\
5 \cdot 4\end{array} \overline{4 \cdot 2}$ & \\
\hline 4. B.subtilis & $\begin{array}{l}a \\
b \\
c\end{array}$ & $\begin{array}{ll}4 \cdot 2 \\
1.0 & \\
2 \cdot 3 & \end{array}$ & $\begin{array}{ll}5 \cdot 8 & \overline{3 \cdot 4} \\
1.0 & \end{array}$ & $\begin{array}{l}4 \cdot 2 \\
1.0\end{array}$ \\
\hline
\end{tabular}

Fig. 2. Part of the composite similarity matrix. Within each box, original similarities are expressed in arbitrary units and then averaged. Similarities taken from: (a) Sneath and Cowan (6), (b) Focht and Lockhart (1), and (c) Goodfellow (2).

tabulated and averaged as before. They were then compared with the three main stems of the phase I dendrogram and added to the dendrogram at the appropriate positions to give a phase II dendrogram (Fig. 4).

The phase I and II dendrograms span the overlapping areas, in terms of OTUs, of the three contributing studies. There now remain those OTUs contained in only one or the other of the three studies, "exclusive OTUs." There were essentially two kinds of exclusive OTU: first, those that in the original studies did show some grouping with other OTUs, either among themselves or with OTUs already contained in the phase I or II dendrograms; second, those that were more or less isolated. The exclusive
OTUs of the first type were added to the phase II dendrogram at those points, in arbitrary units, corresponding to their greatest similarity in the original study. Likewise, the remaining isolated OTUs were added at the most appropriate

\section{Phase I Dendrogram}

\begin{tabular}{lllllllll}
1 & 2 & 3 & 4 & 5 & 6 & 7 & 8 & 9 \\
\hline
\end{tabular}

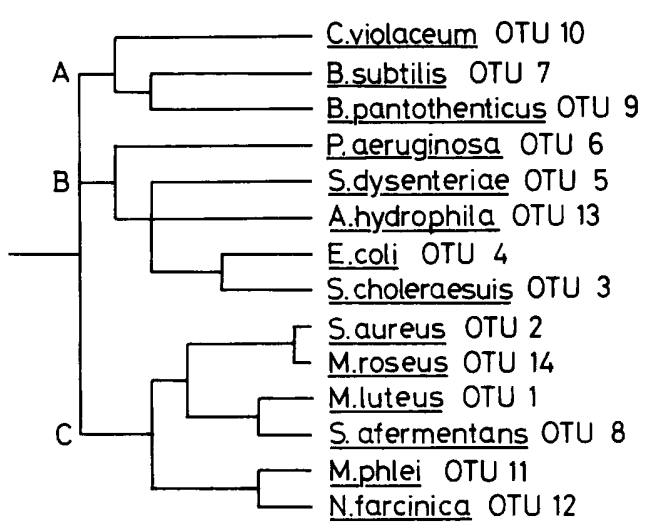

Fig. 3. Phase I dendrogram containing only identifiably identical OTUs common to at least two of the three contributing studies.

Phase II Dendrogram

$\begin{array}{lllllllll}1 & 2 & 3 & 4 & 5 & 6 & 7 & 8 & 9\end{array}$ (Arbitrary scale)

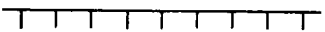

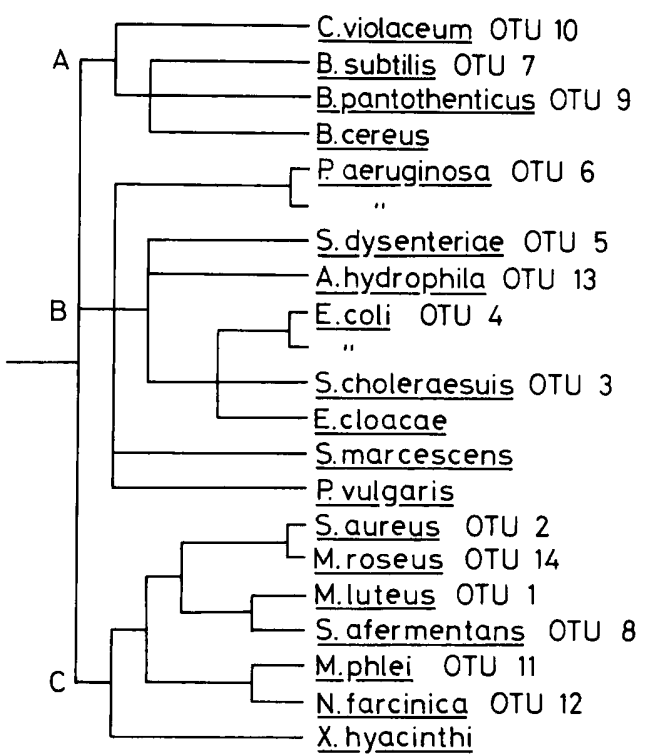

Fig. 4. Phase II dendrogram in which similarly named, but not identifiably identical OTUs, have been added to the phase I dendrogram. 
Phase III Dendrogram

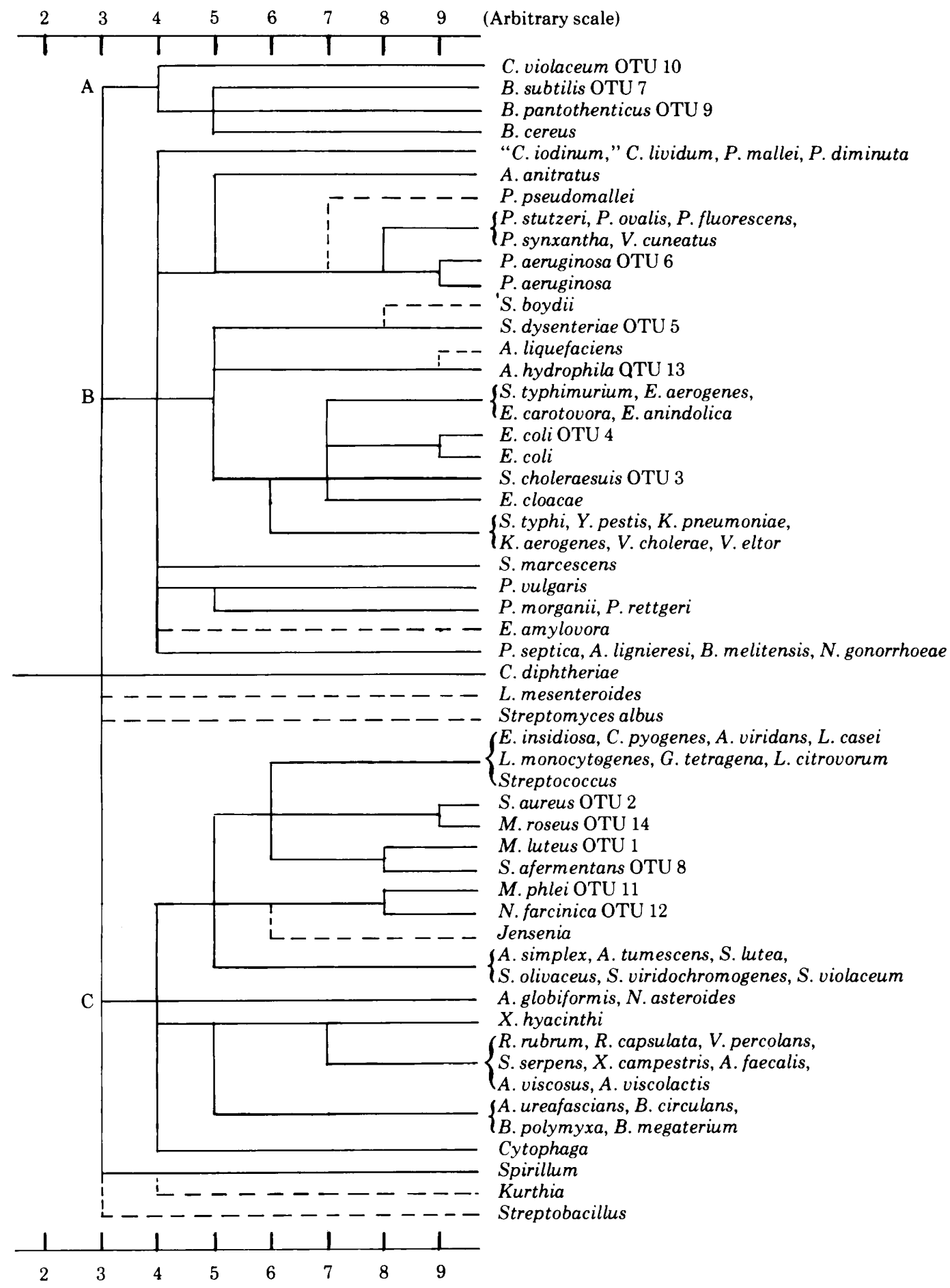

FIG. 5. Phase III dendrogram in which OTUs exclusive to one or other of the contributing studies have been added to the phase II dendrogram. 
points, but as broken lines to indicate more uncertainty.

The final result, a phase III dendrogram, is shown in Fig. 5. It was surprising that few problems arose. In the phase II dendrogram, Xanthomonas hyacinthi appears in a suspect position and in the phase III dendrogram the genus Bacillus has become split into two halves as unrelated, apparently, to each other as to any of the groups. The Bacillus genus, however, illustrates that the real difficulty in combining numerical taxonomies lies, of course, in the exclusive OTUs; the $B$. circulans, $B$. polymyxa, and $B$. megaterium half of the genus was derived from the Goodfellow study and contained not one OTU included in the phase I or II dendrograms.

\section{PROBLEMS OF THE METHOD}

There are other problems with this simple method of interlocking numerical taxonomies, some of which became evident in this first application and others which can be anticipated.

The method cannot handle more than three studies simultaneously, for with more than three studies the composite similarity matrix will have some vacant boxes. If there were not too many of these, the method could still possibly work, although it might need to be supplemented with a method for making educated guesses to fill in empty boxes from the data that are in the matrix.

The contributing studies may use different numerical techniques. This also may not be too great a problem because the many different techniques of numerical taxonomy are surprisingly robust in the sense that they will all (excluding monothetic methods) give the same essential results with one and the same set of experimental data. However, the use of $\% \mathrm{~S}$ coefficients, which exclude negative matches, in one study, and \% $\mathrm{M}$ coefficients, which include negative matches, in another study, will lead to systematic differences and makes standardization of original similarity values necessary, thus introducing a complicating factor in the process. Similarly, some studies use factor analysis of one sort or another without necessarily giving distances (which could be standardized) between OTUs. Again, use of single linkage, as opposed to average linkage, will lead to systematic differences that make a standardization step necessary.

The contributing studies may publish different degrees of detail of their numerical results. For example, the full original similarity matrix may be published with the numerical values or in the form of a shaded triangular matrix, or only the final dendrogram may be published.

Different studies will use different numbers of tests; a priori it may be thought desirable to attach relatively more importance to a study based on more tests than the other studies. (In a preliminary trial of the method, the three contributing studies were, in fact, weighted differently according to the number of tests each had used; this was discontinued when its effect was found to be minimal.) But, although the different studies may list out their tests, often the detailed scoring of each is omitted.

Thus, there are a number of imponderables; study A may be "better" than study B because it was based on more characters, but $B$ may have published more detail than $\mathrm{A}$, and so on.

However, despite these, and doubtless other, difficulties of the present method, if one of the objectives of taxonomy is to derive general classifications, the interlocking of different studies can be regarded as a further step in that direction. A gradual assembling of numerical taxonomies starting with those studies involving only a few species and working upwards (a bottom-to-top strategy) could be done, but here it was elected to begin with the widest possible range of OTUs. This choice was made to try to obviate the criticism mentioned at the beginning. The developed aim would be to see what major groups emerge and then to carry out similar exercises within these, working at lower taxonomic levels.

This method is one way of approaching the general problem of constructing a general classification of bacteria based wholly on numerical taxonomy data. It could be used by a top-tobottom strategy or vice versa. Despite its problems, it has the merit of utilizing further the considerable body of data already available.

\section{ALTERNATIVE APPROACHES TO THE PROBLEM}

One approach would be through the centralized collection of actual test results on OTUs, already being started by V. B. D. Skerman in Australia (5). If such collected data were to be used to derive a single, overall general classification (as opposed to the useful function of data retrieval), then for this approach to be fundamentally an improvement over the method of interlocking numerical taxonomies certain other problems should be resolved first. Of great importance in this context are problems associated with test standardization to improve between-laboratory reproducibility. Also, a high uniformity of test application to diverse organisms would be desirable to achieve a funda- 
mental improvement. This approach depends on good cooperation from individual workers to supply the raw data. It is obviously a very long-term approach and the final job of utilizing the data is one of mammoth proportions.

Another approach would be to ask authors for their transitory numerical data, e.g., actual \%M coefficients, where these have not been published. This would allow the method of interlocking numerical taxonomies to be applied with greater precision, but it again depends on authors' cooperation. Some of the early numerical taxonomy work may be already "dated" to the extent an author may not be willing or able to supply much detail.

Again, another approach would be periodic, new wide-range numerical taxonomies, of the scale of, or only a little larger than, either of the three used in this current application.

Possibly, a combination of the above suggestions would be more practical. This would be: using the existing, published numerical taxonomies to identify centrotypes or median organisms of taxa and possibly some further representatives of taxa to show, with the centrotype, the range of variability of the taxon; initiating centralized data collection on only these privileged strains; and, finally, carrying out new numerical taxonomies on just these strains.

\section{CONCLUSION}

The method of interlocking numerical taxomonies presented here is a semiquantitative, or even crude, approach to a difficult problem. Despite the problems associated with it (which have been discussed), it has brought out a number of ideas and, hopefully, given cause for one to think about the strategic aims of numerical taxonomy as applied in microbiology.

\section{ACKNOWLEDGMENTS}

I thank S. P. Lapage, W. R. Willcox, and I. S. Bowie for helpful discussions.

\section{REPRINT REQUESTS}

Address reprint requests to: Mr. L. R. Hill, National Collection of Type Cultures, Central Public Health Laboratory, London, United Kingdom.

\section{LITERATURE CITED}

1. Focht, D. D., and W. R. Lockhart. 1965. Numeric survey of some bacterial taxa. J. Bacteriol. 90:1314-1319.

2. Goodfellow, M. 1967. Numerical taxonomy of some named bacterial cultures. Can. J. Microbiol. 13:1365-1374.

3. Kubica, G. P., I. Baess, R. E. Gordon, P. A. Jenkins, J. B. G. Kwapinski, C. McDurmont, S. R. Pattyn, H. Saito, V. Silcox, J. L. Stanford, K. Takeya, and M. Tsukamura. 1972. A co-operative numerical analysis of rapidly growing mycobacteria. J. Gen. Microbiol. 73: 55-70.

4. Lockhart, W. R. 1967. Factors affecting reproducibility of numerical classifications. J. Bacteriol. 94:826-831.

5. Skerman, V. B. D. 1973. Statement on the WFCC center for storage, retrieval, and classification of data on microorganisms. Int. J. Syst. Bacteriol. 23:477-479.

6. Sneath, P. H. A., and S. T. Cowan. 1958. An electro-taxonomic survey of bacteria. J. Gen. Microbiol. 19:551-565.

7. Sokal, R. R., and F. J. Rohlf. 1962. The comparison of dendrograms by objective methods. Taxon 11:33-40.

8. Wayne, L. G., T. M. Dietz, C. Gernex-Rieux, P. A. Jenkins, W. Käppler, G. P. Kubica, J. B. G. Kwapinski, G. Meissner, S. R. Pattyn, E. H. Runyon, K. H. Schröder, V. A. Silcox, A. Tacquet, M. Tsukamura, and E. Wolinsky. 1971. A co-operative numerical analysis of scotochromogenic slowly growing mycobacteria. J. Gen. Microbiol. 66:255-271. 